\title{
Evaluating User Experience Elements on a Web System to Support Physiotherapeutic Treatment
}

\author{
Miriéle dos Santos Silvério \\ Instituto Federal de Educação, Ciência e Tecnologia de \\ São Paulo - IFSP \\ R. Antônio Fogaça de Almeida, 200 - Jardim America, \\ Jacareí - SP, 12322-030 \\ miriele.silverio@aluno.ifsp.edu.br
}

\author{
Luciana Brasil Rebelo dos Santos \\ Instituto Federal de Educação, Ciência e Tecnologia de \\ São Paulo - IFSP \\ R. Antônio Fogaça de Almeida, 200 - Jardim America, \\ Jacareí - SP, 12322-030 \\ lurebelo@ifsp.edu.br
}

\begin{abstract}
The trend of process automation has revealed the need of research to include different profile of people to the digital solutions in progress. Therefore, this article is the result of applying aspects of Human-Computer Interaction (HCI) theoretical reference on the developed information system, directed to a physiotherapy clinics. Based on the study of the operation in a specific clinic, we built a web platform that allows the practice of common activities, which used to be done on paper. Hence, the system was evaluated in the production environment to provide the fault finding, referred to the software usability principles, through the user experience. In all, 4 patients and 2 clinic staff participated in the experiment.
\end{abstract}

\section{KEYWORDS}

User Experience, Software Usability, Human-Computer Interaction.

\section{INTRODUÇÃO}

Disfunções na coluna vertebral têm se tornado questões de saúde pública. Segundo estudo realizado por pesquisadores do Global Burden of Disease Study [3], problemas relacionados às colunas lombar e cervical ocupam, respectivamente, o primeiro e quarto lugar no ranque dos distúrbios que mais causam anos de vida perdidos e incapacidade em brasileiros.

No presente, ainda que se desprendam diversos esforços científicos para a compreensão do curso de semelhantes problemas, não há registros de ferramentas automatizadas, que permitam a análise de dados de pacientes com dores crônicas nas costas, e que auxiliem em seu prognóstico evolutivo precoce e tratamento, através da identificação de padrões.

Neste contexto, o projeto de iniciação científica que originou este artigo, tem como objetivo a produção de um sistema de informação, que correlacione informações de tratamento de pacientes, levantando seu histórico de evolução e que através da utilização de técnicas consolidadas em clínicas fisioterapêuticas, possa propiciar a identificação de fatores de risco. Todo esse

In: XVI Workshop de Trabalhos de Iniciação Científica (WTIC 2019), Rio de Janeiro, Brasil. Anais Estendidos do Simpósio Brasileiro de Sistemas Multimídia e Web (WebMedia). Porto Alegre: Sociedade Brasileira de Computação, 2019. (C) 2019 SBC - Sociedade Brasileira de Computação.

ISSN: $2596-1683$ armazenamento era efetuado de forma manual, em fichas no papel.

Os dados armazenados neste sistema serão a base para a análise de técnicas que possam predizer a evolução da dor nos pacientes. Este trabalho é parte de um projeto maior onde pretende-se fazer uma caracterização da evolução dos pacientes com dores nas na coluna vertebral, traçando um perfil dos mesmos e tornando possível a elaboração de um plano de ação mais eficiente.

Dessa forma, o presente artigo tem o objetivo de analisar aspectos importantes do ponto de vista do usuário para verificar sua eficiência. É composto pelas seguintes seções: Sistema Equality, apresenta a solução digital construída para o Grupo Equality clínica de fisioterapia da cidade de São José dos Campos; Referencial Teórico e Metodológico, a qual evidencia os estudos e métricas necessários para a avaliação da interface do sistema proposto; Avaliação de Usabilidade, descreve os processos de avaliação da interface e os resultados obtidos; Trabalhos Relacionados, comparando os trabalhos semelhantes e mostrando suas principais diferenças em relação ao presente trabalho; Conclusão e Trabalhos Futuros, traz as conclusões obtidas no artigo, bem como propostas para trabalhos futuros.

\section{SISTEMA EQUALITY}

Dividido em duas etapas, o projeto encontra-se no momento de implementação do sistema de informação Equality, que possibilita a automatização dos processos manuais desenvolvidos no Grupo Equality. Assim sendo, para que fosse possível o desenvolvimento da plataforma, foram utilizadas as seguintes tecnologias:

- PHP (Hypertext Preprocessor) [7]: Utilizado para o desenvolvimento do lado servidor. Mais especificamente, fora utilizado o framework Laravel [8].

- HTML (Hypertext Markup Language) [9]: Responsável pela estruturação das páginas web.

- $\quad$ CSS (Cascading Style Sheets) [10]: Definiu o estilo das páginas construídas com o auxílio do framework Bootstrap [11].

- JavaScript [12]: Implementado para inserir dinamismo às páginas.

- SQL [13]: Linguagem de consulta padrão dos bancos de dados, empregado através no sistema gerenciador de banco de dados PostgreSQL[14].

Dentre as funcionalidades do sistema construído destacam-se: os cadastros referentes à gestão do sistema, como cadastro de 
fisioterapeutas, especialidades médicas e principalmente de questionários utilizados para avaliação do paciente; o gerenciamento de pacientes; controle de prontuários, funcionalidade que dá acesso as páginas de sessão relacionadas a cada prontuário; e o gerenciamento de sessões de tratamento.

\section{REFERENCIAL TEÓRICO E METODOLÓGICO}

A usabilidade de software está relacionada à facilidade do usuário na interação com a interface, bem como o nível de satisfação que deriva desta ação [1].

A avaliação da usabilidade de um sistema engloba múltiplos aspectos, como: acessibilidade, comunicabilidade, facilidade de aprendizado, facilidade de recordação, dentre outros.

Para a verificação e avaliação desses pontos foram utilizadas métricas obtidas com base na ISO/IEC 9126 [4], a qual é responsável pela padronização de parâmetros de avaliação da qualidade de software. As métricas empregadas são descritas na Tabela 1.

Tabela 1: Métricas de Avaliação de Software

\begin{tabular}{|c|c|}
\hline \multicolumn{2}{|c|}{ Métricas de Avaliação de Produto de Software } \\
\hline Nome e Definição & Método de Avaliação \\
\hline $\begin{array}{c}\text { Atratividade: } \\
\text { O quão atrativa a interface é } \\
\text { para o usuário }\end{array}$ & $\begin{array}{l}\text { Conduza o usuário a testes e } \\
\text { observe o comportamento } \\
\text { dele. } \\
\text { - Fórmula de mensuração: } \\
\text { aplique um questionário de } \\
\text { avaliação da atratividade da } \\
\text { interface para o usuário pós- } \\
\text { uso. } \\
\text { - Método de Avaliação: } \\
\text { Depende do método de } \\
\text { pontuação do questionário. }\end{array}$ \\
\hline $\begin{array}{l}\text { Comunicabilidade: } \\
\text { Facilidade com a qual o } \\
\text { usuário consegue entender as } \\
\text { concepções e intenções do } \\
\text { sistema }\end{array}$ & $\begin{array}{l}\text { Conduza o usuário a testes e } \\
\text { observe o comportamento } \\
\text { dele. Conte o número de } \\
\text { funções que foram } \\
\text { corretamente entendidas e, } \\
\text { compare com o número total } \\
\text { de funções estudadas no } \\
\text { produto. } \\
\text { - Fórmula de mensuração: } \\
\mathbf{x = a} / \mathbf{b} \text { (a: número de funções } \\
\text { entendidas; b: número total } \\
\text { de funções) } \\
\text { - Método de Avaliação: } \\
\mathbf{0}<=\mathbf{x}<=\mathbf{1} \text { (quanto mais } \\
\text { próximo de 1, melhor) }\end{array}$ \\
\hline $\begin{array}{l}\text { Adaptabilidade: } \\
\text { O quão capaz de se adaptar } \\
\text { ao ambiente de operação o } \\
\text { sistema é }\end{array}$ & $\begin{array}{l}\text { Os usuários ou mantenedores } \\
\text { são observados enquanto } \\
\text { tentam adaptar o software ao } \\
\text { ambiente operacional. } \\
\text { - Fórmula de mensuração: } \\
\mathbf{X}=\mathbf{1}-\mathbf{A} / \mathbf{B} \text { (A = Número de } \\
\text { funções cujas tarefas não }\end{array}$ \\
\hline
\end{tabular}

\begin{tabular}{|l|l|}
\hline foram concluídas ou que \\
foram insuficientes para \\
atingir níveis adequados \\
quando comparadas aos \\
testes operacionais com o \\
hardware operacional, B = \\
Número total de funções \\
executadas) \\
Método de Avaliação: \\
Quanto maior, melhor. \\
\hline
\end{tabular}

\section{AVALIAÇÃO DE USABILIDADE}

Nesta seção será descrita a experiência de avaliação das interfaces construídas para o Sistema Equality, a qual foi realizada através da experiência do usuário na utilização da plataforma no ambiente de produção, de acordo com as métricas apresentadas na Seção 3. Esse tipo de experimento foi feito a fim de se obter uma consciência real do padrão de usabilidade desempenhado pelo sistema.

\subsection{Usuários chave}

Os usuários abordados pela avaliação realizada foram funcionários da clínica - os quais possuem interesse em automatizar as tarefas realizadas e têm conhecimento sobre o método de funcionamento dos processos realizados no lugar em questão; e pacientes - aqueles que não possuem conhecimento sobre o funcionamento dos processos desempenhados, visto que, no contexto do Grupo Equality, são estes os maiores interessados no uso do sistema. Ao todo participaram do experimento 6 usuários chave, sendo esses 4 pacientes e 2 funcionários.

\subsection{Metodologia}

O método utilizado para avaliação foi a observação participativa, na qual foram definidas tarefas, de caráter corriqueiro, a serem realizadas por esses usuários. A intervenção do avaliador somente se deu em momentos de solicitação, com o fito de tornar a experiência autêntica.

\subsection{Tarefas e observações}

As tarefas foram realizadas em um dispositivo móvel - tablet, com sistema operacional Android [5]. Este ambiente de operação foi escolhido para teste pois será o de predominância no momento em que o software entrar em operação.

4.3.1 Tarefa 1: Cadastro de um novo questionário. Para realizar esta tarefa o usuário (funcionário da clínica) deveria seguir o seguinte caminho:

1. No menu lateral clicar sobre a opção "Configurações”. Após isso sobre a opção "Questionários”.

2. Clicar sobre o botão com o ícone de “+”, que representaria o cadastro de um novo questionário.

3. Preencher as informações necessárias. Clicar em Salvar. 
Evaluating User Experience Elements on a Web System to Support Physiotherapeutic Treatment

Os resultados obtidos nesse caso mostraram que o usuário apresentou dificuldades quanto ao desenvolvimento dessa tarefa, como: a palavra "Configurações" não deixava clara a função dessa opção; o botão com ícone de "+” não era intuitivo, o usuário não soube onde clicar; haviam muitas etapas para criação de questionário e a relação delas não era clara. Dessa forma, o resultado das métricas analisadas podem ser verificados na Tabela 2.

Tabela 2: Resultado das métricas aplicadas a tarefa 1

\begin{tabular}{|c|c|}
\hline Métrica & Resultados Obtidos \\
\hline Atratividade & $\begin{array}{l}\text { Para avaliação desta métrica foi realizada } \\
\text { uma reunião com os representantes da } \\
\text { clínica, os quais foram responsáveis pelas } \\
\text { sugestões de melhoria, de acordo com a } \\
\text { experiência vivenciada pelos usuários. } \\
\text { Dessa forma, decidiu-se que a opção } \\
\text { "Configurações" seria renomeada para } \\
\text { "Cadastros", e o botão com ícone de “+" se } \\
\text { tornaria "Cadastrar Questionário". }\end{array}$ \\
\hline Comunicabilidade & $\begin{array}{l}\text { Dados de Mensuração: } \\
\qquad \begin{array}{l}\mathrm{a}=2 \quad \mathrm{~b}=3 \mathrm{x}=0,6 \\
\mathbf{1}-\mathbf{x}=\mathbf{0 , 3 3}\end{array} \\
\text { (Grande parte das funções foram } \\
\text { entendidas, no entanto ainda existiam } \\
\text { pontos a serem ajustados) }\end{array}$ \\
\hline
\end{tabular}

As figuras 1,2 e 3 apresentam as interfaces envolvidas nesta tarefa.

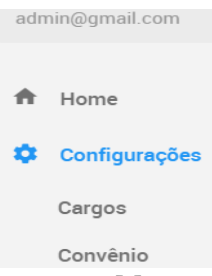

Figura 1: Menu lateral do sistema

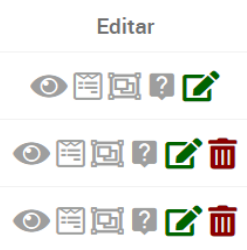

Figura 2: Menu de funções referentes ao questionário

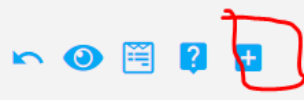

Figura 3: Página de controle de questionários

4.3.2 Tarefa 2: Responder a um questionário. Esta tarefa foi função do paciente, e para realizá-la os seguintes passos foram necessários:

1. Clicar sobre uma das opções de resposta.

2. Clicar no botão "Avançar".

3. Se necessário, e quando for possível, clicar no botão "Voltar" para retornar à questão anterior.

4. Quando a última questão for respondida, clicar sobre o botão "Finalizar".

A partir dessa atividade foram evidenciadas algumas dificuldades, como: a área que evidenciava o número da pergunta em questão não permitia a navegação entre elas; esta mesma área excedia os
Anais Estendidos do WebMedia'2019, Rio de Janeiro, Brasil

limites da tela do tablet, em casos de questionários com maior número de perguntas; os botões "Avançar" e "Voltar" só eram habilitados após a escolha de uma opção de resposta para a pergunta atual, impedindo a navegação livre entre as perguntas. Assim sendo, tornou-se evidente as falhas quanto a adaptabilidade e atratividade da interface, conforme Tabela 3.

Tabela 3: Resultado das métricas aplicadas a tarefa 2

\begin{tabular}{|c|c|}
\hline Métrica & Resultados Obtidos \\
\hline $\begin{array}{c}\text { Adaptabilidad } \\
\mathrm{e}\end{array}$ & $\begin{array}{l}\text { Dados de Mensuração: } \\
\qquad \begin{array}{l}\mathrm{a}=1 \mathrm{~b}=3 \\
\mathbf{1}-\mathbf{1} / \mathbf{3}=\mathbf{0 , 6 6}\end{array} \\
\text { (A medição obtida, ainda que indique mais } 50 \% \\
\text { de êxito de adaptação, mostra que existem pontos } \\
\text { a serem trabalhados para melhor adaptação do } \\
\text { software ao ambiente operacional) }\end{array}$ \\
\hline Atratividade & $\begin{array}{l}\text { Para avaliação desta métrica foi realizada uma } \\
\text { reunião com os representantes da clínica, os } \\
\text { quais foram responsáveis pelas sugestões de } \\
\text { melhoria, de acordo com a experiência } \\
\text { vivenciada pelos usuários. Dessa forma, decidiu- } \\
\text { se que a navegação entre as perguntas deveria ser } \\
\text { livre, visto que, era comum que um paciente } \\
\text { tenha vontade de ler todo o questionário antes de } \\
\text { responde-lo. }\end{array}$ \\
\hline
\end{tabular}

A Figura 4 faz referência aos problemas localizados:

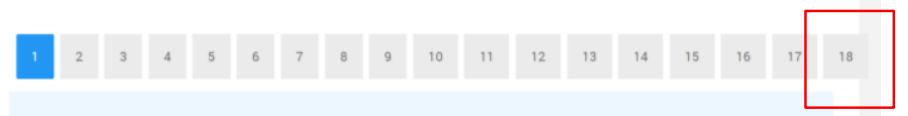

Figura 4: Exemplo do problema de numeração

\subsection{Resultados}

Após as avaliações, através da utilização de métricas e observações da experiência dos usuários chave na utilização do sistema, pôdese desenvolver interfaces cuja usabilidade fora consideravelmente melhorada. Tais interfaces construídas podem ser analisadas através das figuras 5, 6 e 7, nas quais se destacam as seguintes alterações:

- Figuras 5 e 6: Algumas funcionalidades referentes aos questionários deixaram de ser apresentadas como ícones, e passaram a ser abas do processo de criação de questionário; além disso o botão "Cadastrar Questionário" foi implementado. Dessa forma, tornando essa uma tarefa mais fluida.

- $\quad$ Figura 7: Observa-se que o sistema se adapta aos limites de tela e faz quebra de linhas no menu de navegação de perguntas, além disso, a navegação entre essas tornou-se livre 


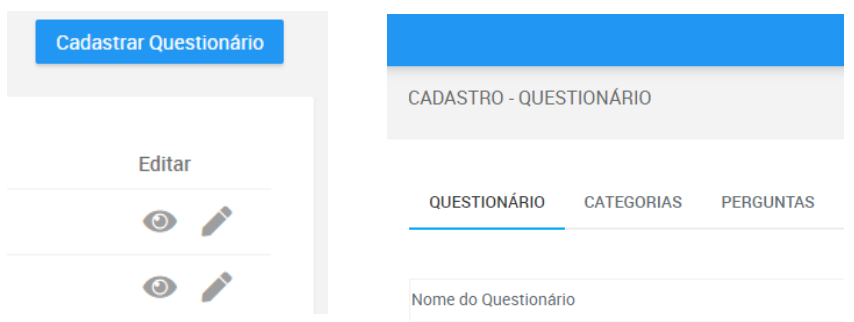

Figura 5: Nova disposição Figura 6: Interface reformulada de funcionalidades de para criação de questionário questionário

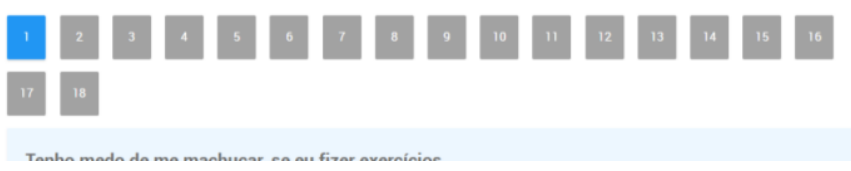

Figura 7: Novo modelo de disposição de número de perguntas

\section{TRABALHOS ELACIONADOS}

Nesta seção apresentam-se revisões breves sobre um trabalho envolvendo avaliação da usabilidade de sistemas da área da saúde. Em [15], os autores usam como método de avaliação métricas estabelecidas pelas ISO/IEC 60601-1-6 e 9126. O objetivo do trabalho realizado foi acompanhar um grupo controlado de usuários chave na realização de tarefas pré-determinadas, a fim de que se apontassem possíveis deficiências de uso do sistema.

Vagner José Lopes, Deborah Ribeiro Carvalho e Janaina Verônica Lahm [16], trabalham com o levantamento, através de pesquisa de satisfação, de fatores problemáticos no uso de interfaces de prontuário eletrônico de pacientes. A escala Likert é utilizada como ferramenta de mensuração de respostas.

O trabalho em [17] analisa fatores de usabilidade no sistema médico BCCT.core. A técnica de avaliação utilizada foi a aplicação de testes de usabilidade, a qual recorre ao uso de questionários aplicados ao usuário com o fito de obter resultados em relação a sua experiência ao utilizar o sistema.

\section{CONCLUSÃO E TRABALHOS FUTUROS}

Neste artigo foi apresentado o processo de avaliação de interfaces do projeto Equality, através do uso de métricas da área de estudo de IHC, em adição a observação da experiência de uso dos usuários chave, a fim de se estabelecer um sistema de fácil utilização, que atenda os diferentes perfis que permeiam a área da saúde. Avaliar a experiência do usuário, seguindo princípios de IHC, foi fundamental para que a plataforma construída apresentasse design atrativo, além de funções claras e intuitivas.

Pretende-se como trabalho futuro a consolidação das métricas utilizadas, com o propósito de se finalizar a etapa de desenvolvimento do sistema de informação, assim, possibilitando o começo da etapa de mineração de dados para gerar diagnóstico preditivo dos pacientes. Em adição, é de interesse desse projeto a continuação da pesquisa quanto a usabilidade e teste de interface, levantando características importantes para o uso do sistema, a fim de que o produto final do projeto se torne uma solução inovadora e de utilização intuitiva. O sistema encontra-se disponível no endereço http://desenvolvimento.projetoequality.com.br.

\section{REFERÊNCIAS}

[1] S.D.J Barbosa e B. S. da Silva (2010). Interação Humano-Computador, (1nd. ed.) . Editora Campus, Rio de Janeiro, RJ, Brasil

[2] W3C, Evaluating Web Sites for Accessibility, https://www.w3.org/WAI/EO/Drafts/impl/eval/.

[3] A disease and injury incidence and prevalence collaborators. global, regional, and national incidence, prevalence, and years lived with disability for 328 diseases and injuries for 195 countries, 1990-2016: a systematic analysis for the global burden of disease study., 390(10100):1211-1259, 2017.

[4] ISO - INTERNATIONAL ORGANIZATION FOR STANDARDIZATION, Systems and software engineering -- Systems and software Quality Requirements and Evaluation (SQuaRE) -- Guide to SQuaRE, 2014.

[5] HISTÓRIA DO ANDROID. ANDROID. Disponível em $<$ https://www.knowledge21.com.br/blog/as-verdadeiras-origens-do-scrum/>. Acesso em 31 jul. 2019.

[6] Sistema Equality. http://desenvolvimento.projetoequality.com.br.

[7] HISTÓRIA DO PHP. PHP. Disponível em <https://www.php.net/manual/pt BR/history.php.php>. Acesso em: 5 jul. 2019

[8] O QUE É LARAVEL? TREINAWEB. Disponível em <https://www.treinaweb.com.br/blog/o-que-e-laravel/>. Acesso em: 5 jul. 2019.

[9] A HISTÓRIA DO HTML. FRONTEND BRASIL. Disponível em <http://www.frontendbrasil.com.br/artigos/a-historia-do-html/>. Acesso em: 5 jul. 2019.

[10] UMA BREVE HISTÓRIA DO CSS. TABLELESS. Disponível em $<$ https://tableless.com.br/uma-breve-historia-do-css/>. Acesso em: 5 jul. 2019.

[11] BOOTSTRAP. GETBOOTSTRAP. Disponível em <https://getbootstrap.com.br/docs/4.1/about/overview/>. Acesso em: 5 jul. 2019.

[12] JAVASCRIPT - UMA BREVE HISTÓRIA DA LINGUAGEM. SHIP IT!. Disponível em <http://shipit.resultadosdigitais.com.br/blog/javascript-1-umabreve-historia-da-linguagem/>. Acesso em: 5 jul. 2019.

[13] SQL - UM POUCO DE HISTÓRIA. INSTITUTO DE FÍSICA. Disponível em <https://www.if.ufrgs.br/ leon/Livro_3 ed/node116.html>. Acesso em: 5 jul. 2019.

[14] THE POSTGRESQL GLOBAL DEVELOPMENT GROUP, PostgreSQL. Disponível em: https://www.postgresql.org/docs/. Acesso em 5 jul. 2019.

[15] SILVA, Barbara Dariano e FILGUEIRAS, Lucia Vilela Leite. Avaliação situada de usabilidade em equipamentos médicos para atendimento à norma IEC 60601-16. Anais do XXVIII Congresso da SBC - WIM Workshop de Informática Médica, Belém do Para - PA, pg. 51-60, 2008.

[16] CARVALHO, Deborah Ribeiro; LAHM, Janaina Veônica e LOPES, Vagner José. KDD na Avaliação da Usabilidade do Prontuário Eletrônico do Paciente por Profissionais da Enfermagem. Revista Brasileira de Inovação Tecnológica em Saúde, v. 6, n. 3, 2016.

[17] AFONSO, Ana Paula; CARDOSO, Jaime S.; CARDOSO, Maria João e COTA, Manuel Pérez. A Avaliação da Usabilidade de um Sistema Médico Inteligente: BCCT.core. $5^{\text {a }}$ Conferência Ibérica de Sistemas e Tecnologias de Informação, Póvoa do Varzim, Portugal, v. 1, 2009.

\section{AGRADECIMENTOS}

Ao CNPq (Conselho Nacional de Desenvolvimento Científico e Tecnológico), pelo apoio financeiro por meio da bolsa do Programa Institucional de Bolsas de Iniciação em Desenvolvimento Tecnológico e Inovação (PIBITI) e ao IFSP, pela disponibilização da infraestrutura para realização desta pesquisa. 